\title{
Influence of shear-induced crystallization on the
}

\section{electrical conductivity of high density polyethylene}

\section{carbon nanotube nanocomposites}

${ }^{1}$ Fangfang Tao*, ${ }^{2}$ Leïla Bonnaud, ${ }^{1}$ Dietmar Auhl, ${ }^{3}$ Bernd Struth, ${ }^{2}$ Philippe Dubois, ${ }^{1}$ Christian Bailly*

${ }^{1}$ Bio- and Soft Matter, Institute of Condensed Matter and Nanosciences, Université catholique de Louvain, B-1348 Louvain-La-Neuve, Belgium

${ }^{2}$ Laboratory of Polymeric and Composite Materials, Center of Innovation and Research in Materials \& Polymers (CIRMAP), Materia Nova Research Center \& University of Mons, 20 Place du Parc, 7000 - Mons, Belgium

${ }^{3}$ DESY, Notkestrasse 85, D-22607 Hamburg, Germany

*Corresponding author: fangfangtao.nju@gmail.com; christian.bailly@uclouvain.be

\begin{abstract}
:
The relation between shear mixing time in the melt, polymer crystal size, and electrical conductivity is studied for high density polyethylene - carbon nanotubes nanocomposites with
\end{abstract}


the help of in-situ rheo-dielectric and rheo-small angle synchrotron X-ray scattering (SAXS) techniques. Results show that the memory of crystal structure obtained after melt-mixing is not easily removed by re-melting and annealing. The conductivity of composites shear-mixed for 20 min. cannot reach that of $10 \mathrm{~min}$. samples, even after additional quiescent annealing for $6000 \mathrm{~s}$ at $190^{\circ} \mathrm{C}$. The rheo-SAXS data further indicate that the temperature needed to melt all the crystals is higher for the longer time shear mixed composites. These results all suggest that with long processing time, larger crystals are nucleated and grow on the nanotubes, which reduce electrical conductivity, presumably because they prevent electrical contact between the nanotubes.

\section{Introduction}

Nanocomposites of polyethylene (PE) and carbon nanotubes (CNT) have attracted a lot of academic and industrial interest due to their unique mechanical and/or electrical properties[1-7]. The dispersion of pristine CNT in polyolefins by melt-mixing is usually very poor[5, 7-12], due to weak interaction between polymer chains and CNT as well as the strong van der Waals interaction between CNT. To ensure a good load transfer from the CNT to the PE matrix as well as a good dispersion of the nanofiller, surface modification of CNT is used in several reports[6, 13]. For example, PE-grafted CNT can be used to spectacularly reinforce the mechanical properties of a HDPE matrix[6]. Recently, Dubois et al. have developed a new method derived from a polymerization-filling technique[13]. A highly active metallocene complex is grafted to the CNT surface and polyethylene chains grow from there. However CNT chemical modification is complex and can be detrimental to mechanical or electrical properties. Therefore direct mixing of pristine CNT with PE is usually more attractive for applications[14-17]. The HDPE matrix conformation is strongly influenced at the interface with CNT as the latter are very effective nucleators of HDPE crystallization. A well-known shish-kebab structure, in which CNT act as 
shish and HDPE lamellae acts as kebab, can be formed during either solution[18, 19] or melt mixing[20]. Besides, an extended-chain layer of HDPE aligning at the surface of CNT is also mentioned[21]. In studies by Fu et al.[22], such a layer is found when using dynamic packing injection molding (DPIM) and it can disappear after heating to $170^{\circ} \mathrm{C}$. Minus et al. however[21], observe that single-wall CNT templated PE kebab crystals and the extended layers do not redissolve in boiling xylene. Moreover, the authors find that the chain conformation becomes disorganized upon melting but remains strongly oriented along the nanotube axis. In general, the question regarding the shish-kebab structure of HDPE crystals growing around CNT still remains unclear.

Processing-related crystalline structure influences electrical conductivity by disturbing the CNT conductive network near the percolation threshold of HDPE/CNT nanocomposites. A review study on the dispersion of pristine CNT and the electrical conductivity of HDPE/CNT composites has recently been published by some of the authors[11]. It appears that every combination of HDPE and CNT leads to its own result in terms of morphology (i.e. extent of nanofiller dispersion), electrical conductivity and thermal properties. Therefore, it is very hazardous to predict the electrical conductivity of a given HDPE/CNT composite based on general considerations. Besides, the influence of melt-processing parameters such as screw configuration, rotation speed, mixing time and temperature on the morphology and conductivity of the CNT-based composites is well-studied in literature[8, 9, 12, 14, 23-30]. More specifically, specific mechanical energy (SME) increases with rotation speed, lower melt temperature and reduced throughput, whereas best CNT dispersion was found at highest SME. However, too high SME (i.e. shear stress) may lead to a decrease of CNT aspect ratio, and hence increase of resistivity[31-34]. For example, it was recently shown and quantified by Krause et al.[33, 34] 
that melt processing of CNT-polymer composites can lead to significant CNT shortening, which influences the properties of the composites. On the other hand, the influence of processing related crystalline structure on electrical conductivity has been rarely studied. However its importance cannot be neglected since processing can disturb the CNT conductive network near the percolation threshold, especially taking into account that shear deformation is known to play an important role in PE crystallization[22, 35, 36]. The formation of shish-kebab structures around CNT can have a two-sided effect. On the one hand, it is positive for mechanical properties[22, 37]; on the other hand, it should be negative for electrical properties since the crystals can push the CNT away from each other as well as block the electrons from hopping between CNT[23].

In this work, we study two HDPE/CNT nanocomposites with exactly the same components but different shear-mixing times in the melt. We find that electrical properties of the final nanocomposites are greatly influenced by the pre-mixing history in melt state and that the origin of this effect is not a straightforward CNT length reduction but is closely related to the HDPE crstallization behavior. To study the nucleation and growth mechanisms of HDPE crystals in the presence of CNT, we investigate the crystallization and re-melting behavior by in-situ smallangle synchrotron X-ray scattering combined with shear rheology (Rheo-SAXS). The meltmixing time, HDPE crystal size, and final electrical conductivity are found to be strongly interconnected.

\section{Experimental}

\section{Materials}

The CNT used in this study are provided by FutureCarbon. They are thin homogeneous tubes with 10-20 concentrically bent single graphene layers with an average diameter of $\sim 20 \mathrm{~nm}$ and 
purity of $>98 \%$.

A high and a low viscosity Ziegler-Natta HDPE samples are used for the matrix, respectively MS201 BN-NA from Total Petrochemicals (referred to as high molecular weight HDPE, "HMW-PE” for short ) and Lacqtene HD 2040 ML 55 from Arkema (referred to as low molecular weight HDPE, “LMW-PE” for short ). The molar mass distribution of the samples has been inferred from rheology as described in the results section. The composite samples blended with CNT are referenced as HCXX or LCXX with "H” and "L” referring to "high" and "low" molar mass samples respectively, "C" stands for "CNT", and XX is the shear mixing time (see Table 1).

\section{Melt compounding and sample preparation}

An internal mixer (Brabender, Germany) is used to prepare all composites. For melt mixing experiments, the conditions are as follows: $190^{\circ} \mathrm{C}, 3$ minutes at $30 \mathrm{rpm}$ (for introduction of materials) and further 7 minutes or 17 minutes, respectively, at $60 \mathrm{rpm}$.

Table 1 Information of sample preparation

\begin{tabular}{|c|c|c|c|}
\hline Samples & $\begin{array}{l}\text { Mixing time in } \\
\text { brabender (min) }\end{array}$ & Composition & MFI of the HDPE matrix \\
\hline HC10 & 10 & 4.6\% CNT+HMW-PE & $\begin{array}{l}\text { No flow } / 10 \mathrm{~min} \text { at } 2.16 \mathrm{~kg} \\
8.0 \mathrm{~g} / 10 \mathrm{~min} \text { at } 21.6 \mathrm{~kg}\end{array}$ \\
\hline HC20 & 20 & 4.6\% CNT+HMW-PE & -- \\
\hline LC10 & 10 & 1\% CNT+ LMW-PE & $20 \mathrm{~g} / 10 \mathrm{~min}$ at $2.16 \mathrm{~kg}$ \\
\hline LC20 & 20 & 1\% CNT+ LMW-PE & -- \\
\hline
\end{tabular}

For further characterization of electrical, rheo-dielectrical and rheo-SAXS properties, the samples are compression molded after extrusion. The same protocol is followed for each sample. 
An AGILA PE ZO Hot Press is used to mold the samples. Pieces of composite samples are placed in the square frames $(5 \mathrm{~cm} * 5 \mathrm{~cm})$ of a steel plate. The molding program has three stages: first, hot pressing at low pressure (10 bars) for $3 \mathrm{~min} 20 \mathrm{sec}$ following with 3 degassing steps at 90 bars, 110 bars, and 150 bars respectively; second, hot pressing at high pressure (150 bars) for $3 \mathrm{~min}$; finally, cold pressing at room temperature and 80 bars for 3 min. The products are used for electrical tests at room temperature. A second molding is performed on the samples to press them into a thin disc shape. The molding program is the same as the first one, and the disc-like samples are of $100 \mu \mathrm{m}$ thick for rheo-dielectric and 500 $\mu \mathrm{m}$ thick for rheo-SAXS tests.

\section{Soxhlet extraction}

A Soxhlet device has been used to extract the CNT from composites HC10 and HC20. Samples weighing $50 \mathrm{mg}$ are deposited in a glass fibre thimble. The extraction is performed using p-xylene at $140^{\circ} \mathrm{C}$ for 5 hours. Next, the CNT suspended in p-xylene solvent are deposited on 300 mesh holey-carbon copper grids and observed under TEM to study the crystal structure of HDPE.

\section{Characterization}

\section{High Resistance Electrometry}

For samples with a resistivity higher than $10^{6} \mathrm{ohm} . \mathrm{cm}$, the measurement is performed on $8 \times 10$ $\mathrm{cm}^{2}$ and $3 \mathrm{~mm}$ thick plates with an electrometer (6517B Keithley) combined with a 8009 box

from Keithley. For more conductive samples, measurements are performed on three 1x6x0.3 cm bars using a Keithley multimeter 2700. Silver paint is coated on the surface of the samples to minimize the contact resistance.

Thermogravimetry Analysis (TGA) 
TGA measurements are carried out with a Mettler Toledo TGA/SDTA 851e at an air flow of $50 \mathrm{~cm}^{3} / \mathrm{min}$. The following thermal program is applied to the HDPE/CNT systems in order to remove the HDPE matrix: Temperature is increased from $25^{\circ} \mathrm{C}$ to $550^{\circ} \mathrm{C}$ at a heating rate of $20^{\circ} \mathrm{C} / \mathrm{min}$. The corresponding weight loss is $93 \mathrm{wt} \%$ for both HC10 and HC20. Considering that the weight loss for neat CNT is about $5 \mathrm{wt} \%$, we speculate that HDPE is not fully degraded. Nevertheless, after this treatment, most of the CNT are isolated from the HDPE matrix and can be well-dispersed in chloroform solution. A drop of this CNT suspension is then placed on holey-carbon copper grids. After evaporation of the solvent, the CNT remain on the grids and their length can be measured by TEM.

\section{Transmission electron microscopy (TEM)}

The specimens are cut using a Reichert Microtome. Ultrathin sections of approximately 70-100 $\mathrm{nm}$ in thickness are cut using a cryodiamond knife (Diatome, Switzerland) and collected on 400 mesh copper grids. The CNT dispersion in nanocomposites is investigated using a LEO 922 Transmission Electron Microscope operating at 200kV. The nanotube lengths are measured on approximately 1000 particles applying the software analySIS ${ }^{\circledR} 3.2$ (Olympus Soft Imaging Solutions GmbH, Germany) using the full visible length of each separated nanotube not touching the edge of the image or entangled to each other by applying the polyline function.

\section{Differential scanning calorimetry (DSC)}

The non-isothermal crystallization of all nanocomposites is analyzed under nitrogen with a DSC 821e differential scanning calorimeter (Mettler-Toledo, Switzerland). The temperature is calibrated with Indium and Zinc. Samples of about $5 \mathrm{mg}$ are first heated from room temperature to $190^{\circ} \mathrm{C}$ (1st heating) at $10^{\circ} \mathrm{C} / \mathrm{min}$ and kept at this temperature for $5 \mathrm{~min}$. Next, the samples are 
cooled down to room temperature at a constant cooling rate of $-10{ }^{\circ} \mathrm{C} / \mathrm{min}$, and finally heated back to $190^{\circ} \mathrm{C}$ (2nd heating).

\section{Rheo-Dielectrics}

The rheometer used is Physica MCR-301 (Anton Paar, Austria) with a special upper rotating geometry having an isolating ceramic layer and a bottom steel plate being separated from the instrument by a PEEK support. Four wires connected to the upper and lower plate are separately connected via BNC ports with the impedance analyzer HP-4284A (Hewlett Packard, United States). The alternating current (AC) conductivity is measured in the frequency range of $20 \mathrm{~Hz}$ to $1 \mathrm{MHz}$ using a voltage of $5 \mathrm{mV}$. Dynamic rheological properties of the samples are measured with a $8 \mathrm{~mm}$ diameter parallel plate geometry at $150^{\circ} \mathrm{C}$. Frequency sweeps from 628 to $0.02 \mathrm{rad} / \mathrm{s}$ are applied at a strain of 1\% (linear viscoelastic region). The sample disks with a thickness of 0.2 mm are prepared by compression molding. The dependence of complex viscosities $\eta^{*}$, storage modulus G' and loss modulus G', on the oscillatory frequency $\omega$ is determined by frequency sweeps (Figure 1).

The zero-shear viscosity of neat high molar mass HDPE is not reached in the measured frequency range, indicating some very long relaxation times of the HDPE matrix chains. In the whole frequency region, the $\eta^{*}$ and $\mathrm{G}^{\prime}$ of HC10 and HC20 are higher than those of neat HDPE, which is caused by the addition of CNT. 


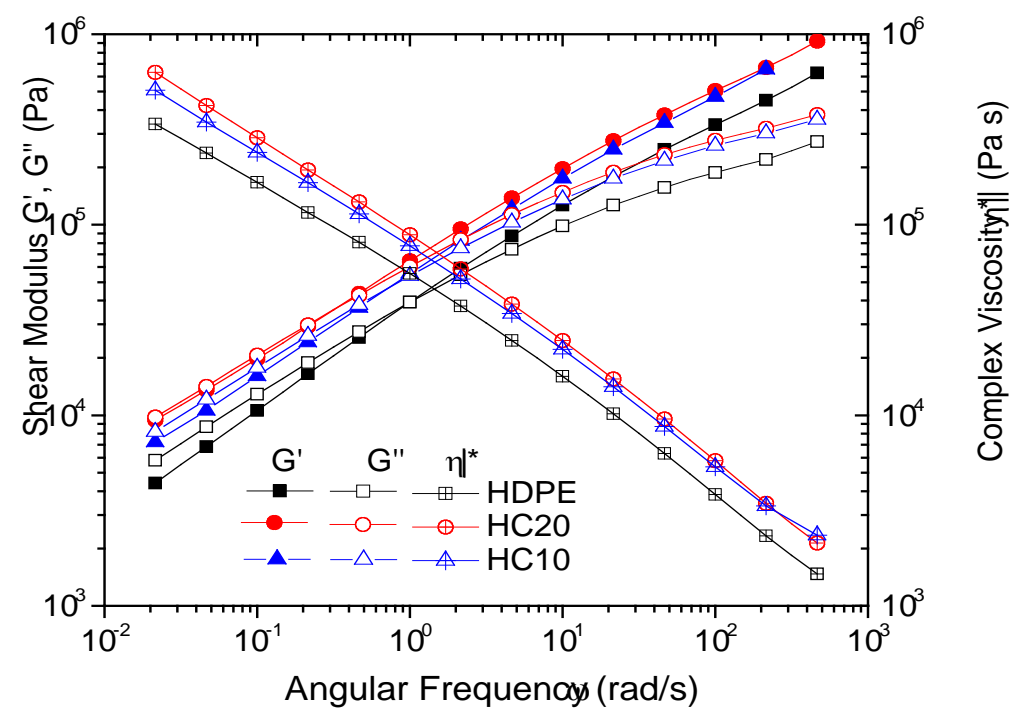

Figure 1. Complex viscosity $\eta^{*}$ as well as storage G' and loss modulus G’’of HDPE, HC10 and HC20 at $150{ }^{\circ} \mathrm{C}$.

\section{Rheo-SAXS}

In-situ Rheo-SAXS measurements are carried out at the beamline BW1 of the DORIS III storage ring at HASYLAB (DESY, Hamburg, Germany). The selected wavelength is $0.12589 \mathrm{~nm}$. A specifically modified stress-controlled rheometer (Mars II, Thermo Haake Scientific) is used with a custom-made polymeric (Kapton, Vespel®) plate-plate geometry. These geometries have thin windows of $0.3 \mathrm{~mm}$ thickness to minimize scattering and absorption of the beam. The rheometer is equipped with a custom-made cylindrical heating cell operating under nitrogen to avoid oxidation of the polymer melts as well as to keep the temperature homogeneity inside the oven. The temperature accuracy is of $\pm 0.5^{\circ} \mathrm{C}$. A diamond reflector is used to redirect the intrinsically horizontal synchrotron X-ray beam by $90^{\circ}$. The scattered intensities are recorded by a 2D detector (Pilatus 100k) positioned at a distance of $2.74 \mathrm{~m}$ above the sample. The data are evaluated by the Fit2D software (ESRF Grenoble, France). All images are normalized for beam 
fluctuations and sample absorption. The scattering intensity is integrated for all azimuthal angles, assuming there is very little shish structure in the system.

HC10 and HC20 samples are studied following an identical temperature program (cf. inset in Fig. 7) with loading a sample of about $0.5 \mathrm{~mm}$ thickness at room temperature and heating to $190^{\circ} \mathrm{C}$ with a rate of about $15^{\circ} \mathrm{C} / \mathrm{min}$ (marked as “ $1 \mathrm{~h}$ ”), followed by cooling to $120^{\circ} \mathrm{C}$ with a rate of about $-12^{\circ} \mathrm{C} / \mathrm{min}$ (“1c") and repeating these steps.

\section{Results and discussion}

The melt-rheological data are discussed in further detail in Figure 2 since the longest relaxation times are found to play an important role for the materials behavior and interpretation. The overlaid creep and oscillatory shear data are fitted by the tube-theory based time-marching algorithm of Van Ruymbeke et al.[38, 39], which is able to describe the rheological response of HDPE and other polydisperse polymer melts. From previous studies[40] on HDPE the characteristic model parameters are fixed, e.g. the rubber-elastic plateau modulus $G_{\mathrm{N}}{ }^{0}=4 / 5 G_{\mathrm{e}}=$ 2.1 MPa, entanglement relaxation time $\tau_{\mathrm{e}}=2.5 \mathrm{~ns}$, and molecular weight between entanglements $M_{\mathrm{e}}=1500 \mathrm{~g} / \mathrm{mol}$. Thus, these material parameters can be used to determine the molecular weight distribution (MWD) from rheological data by matching the relaxation time distribution via an inverse modeling procedure.

For the MWD of the pure PE materials LMW-PE and HMW-PE an assumed single and bimodal log-normal shape, respectively, was used to best match the experimental linearviscoelastic data, and a special emphasis was dedicated to the phase angle, the high sensitivity of which is highlighted in figure 2 (right). The LMW-PE was found to have an average molecular weight of about $220 \mathrm{~kg} / \mathrm{mol}$ with a polydispersity of 4, whereas the HMW-PE shows a significantly higher average molecular weight of about $890 \mathrm{~kg} / \mathrm{mol}$ and polydispersity of 16 , 
respectively, due to a significant HMW fraction. The longest relaxation time of the HMW one is estimated to be more than $10^{4} \mathrm{~s}$.
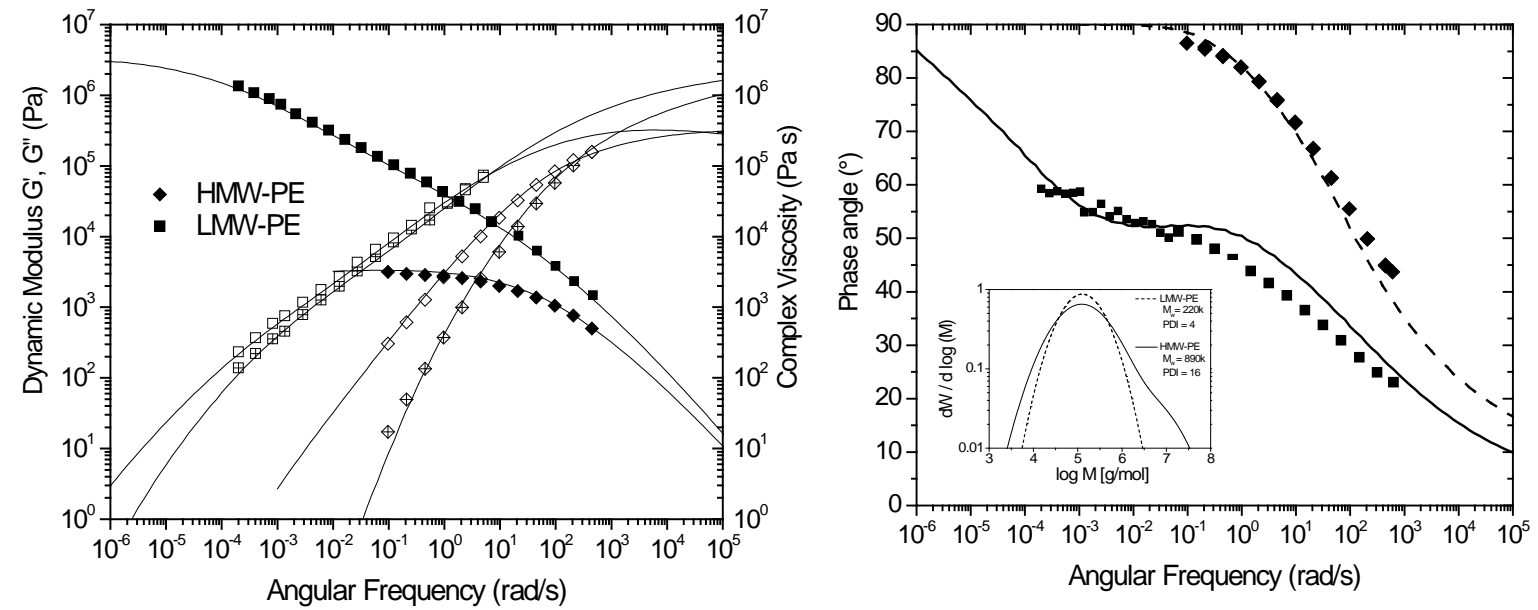

Figure 2. Complex viscosity and dynamic moduli as well as phase angle versus angular frequency for all samples at $150{ }^{\circ} \mathrm{C}$ from creep and oscillation as well as molecular modeling.
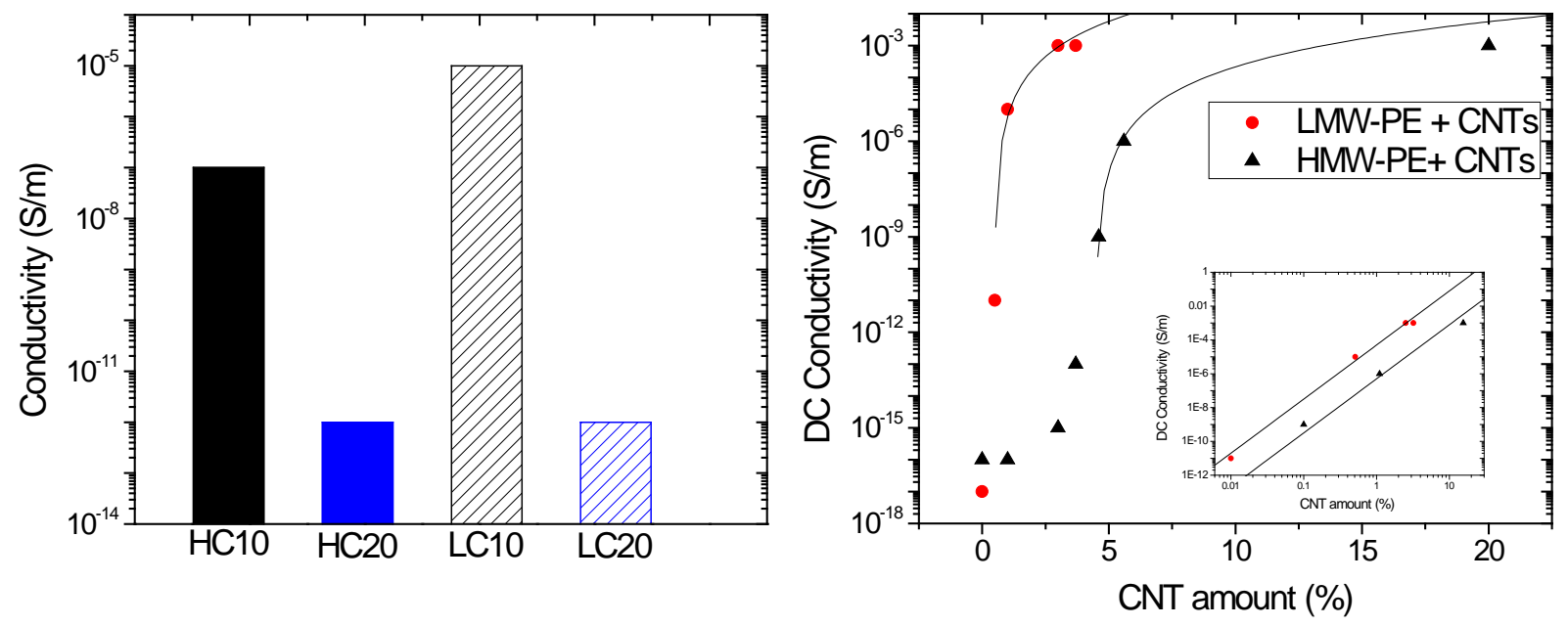

Figure 3. DC Conductivity of compression-molded composites at room temperature. Comparation of conductivity for composites with 10 min or 20 min mixing time (left); Conductivity versus CNT concentration with 10 min mixing (right). 
The compositions of the four samples listed in Table 1 are carefully chosen to be around the percolation threshold of the specific system, in order to highlight the effects of processing time on the final conductivity. Figure 3(right) shows the change of Direct Current (DC) conductivity with the weight fraction of CNTs in the final hot-pressed nanocomposites at room temperature. In order to get an estimate for percolation concentration $\left(p_{c}\right)$, we fit the experimental DC data to the following equations:

$$
\sigma_{D C} \propto\left(p-p_{c}\right)^{t}, \quad \text { for } p>p_{c}
$$

Which represent the well know scaling law of the composite conductivity near the percolation threshold[41].The solid lines on figure 3 are calculated from equation (1) using the fit values of $p_{c}, t$ and $s$. The best linear fit for DC vs. $\left(p-p_{c}\right)$ data on a log-log scale are found at the percolation concentration $p_{c}$ about $0.49 \mathrm{wt} \%$ and critical exponent $\mathrm{t}=3.5$ for CNTs/LMW-PE system blended for $10 \mathrm{~min}$, while $p_{c}$ about $4.5 \mathrm{wt} \%$ and critical exponent $\mathrm{t}=3.5$ for CNTs/HMW-PE system blended for 10 min (see inset in figure 3 (right)).

In figure 3(left), HC10 and LC10 have widely different DC conductivity values from HC20 and LC20 at room temperature. After 10 min of mixing and following compression-molding, the conductivity of HC10 and LC10 is $10^{-7} \mathrm{~S} / \mathrm{m}$ and $10^{-5} \mathrm{~S} / \mathrm{m}$, respectively. However, after 20 min of mixing, the conductivity of HC20 and LC20 decreases to $10^{-12} \mathrm{~S} / \mathrm{m}$.

Three major explanations can be considered for the loss of conductivity after a long mixing time:

1) The CNT are shortened during long time mixing, which leads to a lower aspect ratio of filler particles and therefore a loss of conductive network. There are several literature reports regarding the influence of melt-mixing time on the final CNT length[12, 26, 32, 33, 42]. It is frequently invoked to explain loss of conductivity in literature. 
2) The morphology of the composites, i.e. the dispersion state of CNT is changed, thus altering the (average) distance between CNT.

3) The crystal structure of HDPE has been altered due to strong mixing forces and alignment of molecules.

In order to examine these possibilities, the HC10 and HC20 samples are investigated in detail. To avoid duplication, we only discuss composites of HWM-PE with CNT, which is sufficient to demonstrate our point. First, the CNT length distribution is studied using TEM, based on the method proposed in Krause et al.[32]. In Figure 4, the inserted TEM pictures show that the CNT are well separated. To quantify the CNT length distribution, the typical distribution parameters $\mathrm{x}_{10}, \mathrm{x}_{50}$ and $\mathrm{x}_{90}$ are calculated indicating that $10 \%, 50 \%$, and $90 \%$ of the CNT lengths are smaller than the given value. These values are listed in Table 2. The CNT length reduction is about 2.3\% (related to $\mathrm{x}_{50}$ ) when comparing $\mathrm{HC} 10$ and HC20, which is not significant and the dramatic loss of conductivity cannot be attributed to this. As a consequence, the first possibility considering the damage on CNT length as the main explanation for the loss of electrical conductivity is ruled out. However, since conductivity is extremely sensitive to a change of CNT length in the vicinity of the percolation threshold, the slightly shorter CNT at longer processing time could still play a minor role in decreasing conductivity.

Table 2. Calculated value of distribution parameters $\mathrm{x}_{10}, \mathrm{x}_{50}$ and $\mathrm{x}_{90}$

\begin{tabular}{llll}
\hline Samples & $\mathbf{x}_{\mathbf{1 0}}(\mu \mathrm{m})$ & $\mathbf{x}_{\mathbf{5 0}}(\mu \mathrm{m})$ & $\mathbf{x}_{\mathbf{9 0}}(\mu \mathrm{m})$ \\
HC10 & 0.490 & 1.044 & 2.121 \\
HC20 & 0.480 & 1.020 & 1.986 \\
\hline
\end{tabular}



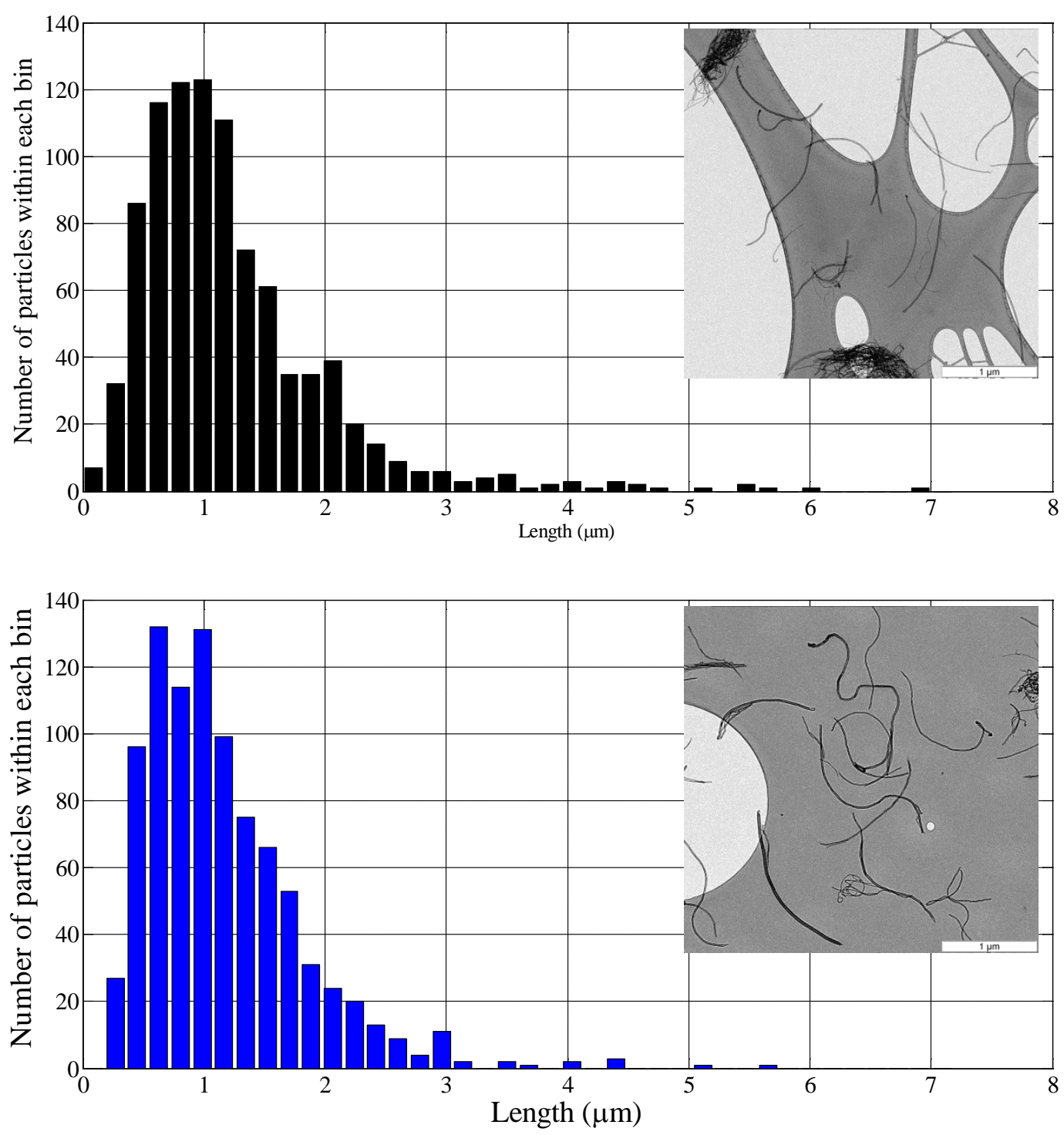

Figure 4. Length distribution of CNT in HC10 (upper) and HC20 (lower). The background of the insert TEM pictures is from holey-carbon films of the copper grids.

In the search for clues to confirm or infirm the second assumption, morphology of the HC10 and HC20 composites right after compression molding is further investigated using TEM. From Figure 5(a) and 5(b), we clearly see that CNT dispersion in HC10 and HC20 is quite similar, stressing that longer melt-mixing time does not lead to a significant change. More precisely, in both figures 5(c) and (d), the size of the agglomerates is about $0.5-1 \mu \mathrm{m}$. Very few isolated CNT 
are observed around the agglomerates and big lamellar crystals of HDPE are also found. As a consequence, evolution of CNT dispersion state during processing cannot explain the observed electrical conductivity change.

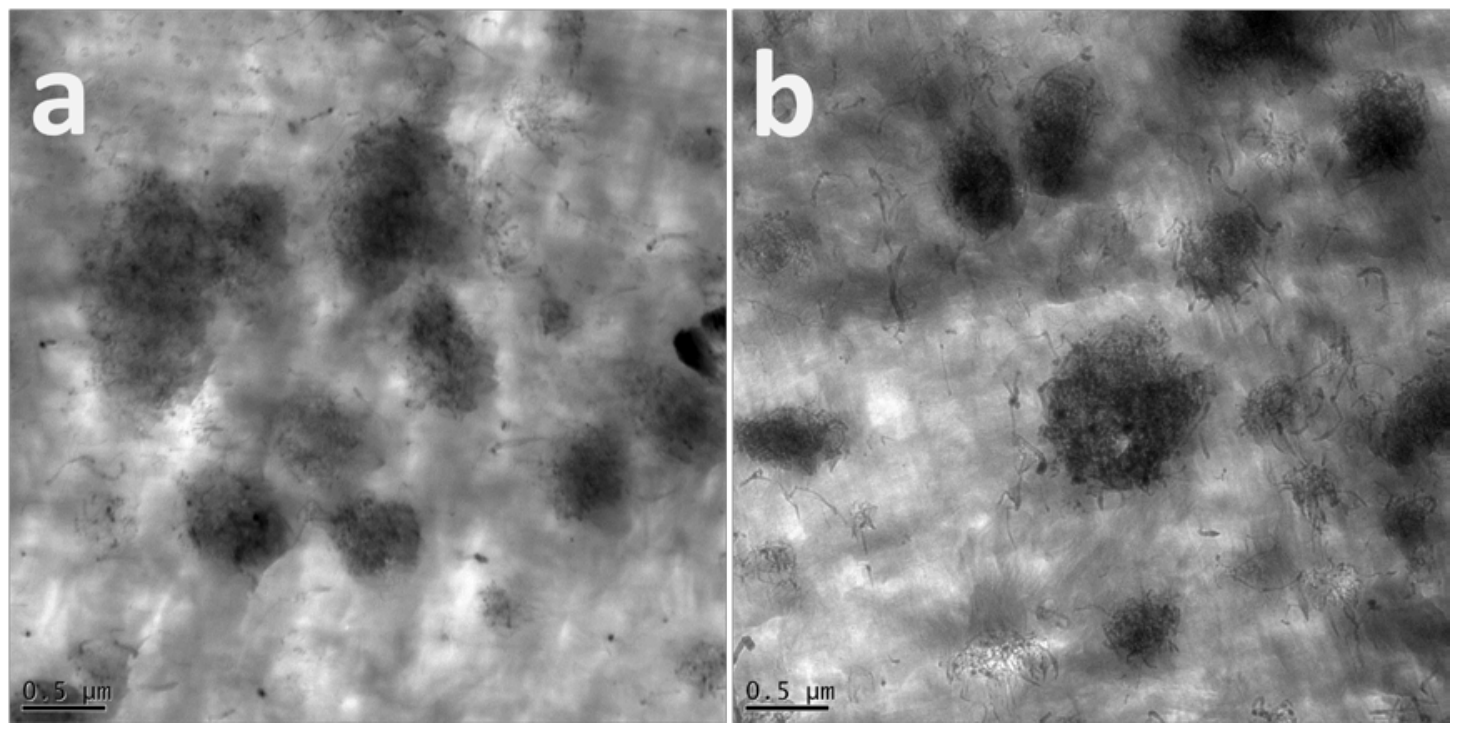

Figure 5. TEM morphology of HC10 (a), and HC20 (b), right after compression molding.

To explain the conductivity difference between HC10 and HC20, the third assumption left to be checked is the possible influence of processing time on resulting HDPE crystal structure. For that purpose, in-situ rheo-dielectrical and rheo-SAXS are used to track the melting and crystallization of HDPE and their influence on the conductivity of the composites.

The values at a frequency of $100 \mathrm{~Hz}$ are taken as DC conductivity are shown versus time in the melt state in figure 6. For the same amount of CNT and identical HDPE matrix, the conductivity of HC10 and HC20 versus time follows different trends. The big filled squares at start time are the conductivity of HC10 and HC20 at room temperature.

In HC10, the increase of DC conductivity during annealing at $190^{\circ} \mathrm{C}$ for $6000 \mathrm{~s}$ can be explained by melting of the HDPE crystals and rebuilding of the CNT conductive network. The 
CNT which are oriented, separated and distorted during mixing and compression-molding tend to reform contacts during quiescent annealing in the melt (secondary agglomeration). The driving force can be Van der Waals interactions between CNT and/or depletion effects between CNT and polymer chains. Next, the observed decrease of conductivity by about half a decade when the sample is subsequently cooled to $125^{\circ} \mathrm{C}$ is caused by matrix crystallization. The crystals grow from the surface of the CNT and drive them apart. Moreover, the crystalline part can also isolate and trap electrons[23], which reduces conductivity. For HC20, the change of conductivity versus time follows a different trend. The DC conductivity increases faster in HC20 compared to that of HC10 and reaches $10^{-6} \mathrm{~S} / \mathrm{m}$ only after about $1000 \mathrm{~s}$ of annealing in melt. The conductivity decreases by more than one order of magnitude in the range of $125^{\circ} \mathrm{C}$ during recrystallization. The decrease of conductivity caused by the crystals growth from the surface of CNT is more pronounced than for HC10, although the crystallinity of HC10 and HC20 are similar around 70\%. This suggests that the crystal area of HC20 is larger than that of HC10, and hence pushing the CNT further apart and/or better blocking electrons.

After 4 loops of annealing, cooling and heating, the conductivities of $\mathrm{HC} 10$ and $\mathrm{HC} 20$ at $50^{\circ} \mathrm{C}$ (could be referred as room temperature since there is no further crystallization) are approaching each other. This suggests that HDPE crystals re-arrange and finally reach a similar crystal size after a sufficient long time of annealing and re-crystallization. 


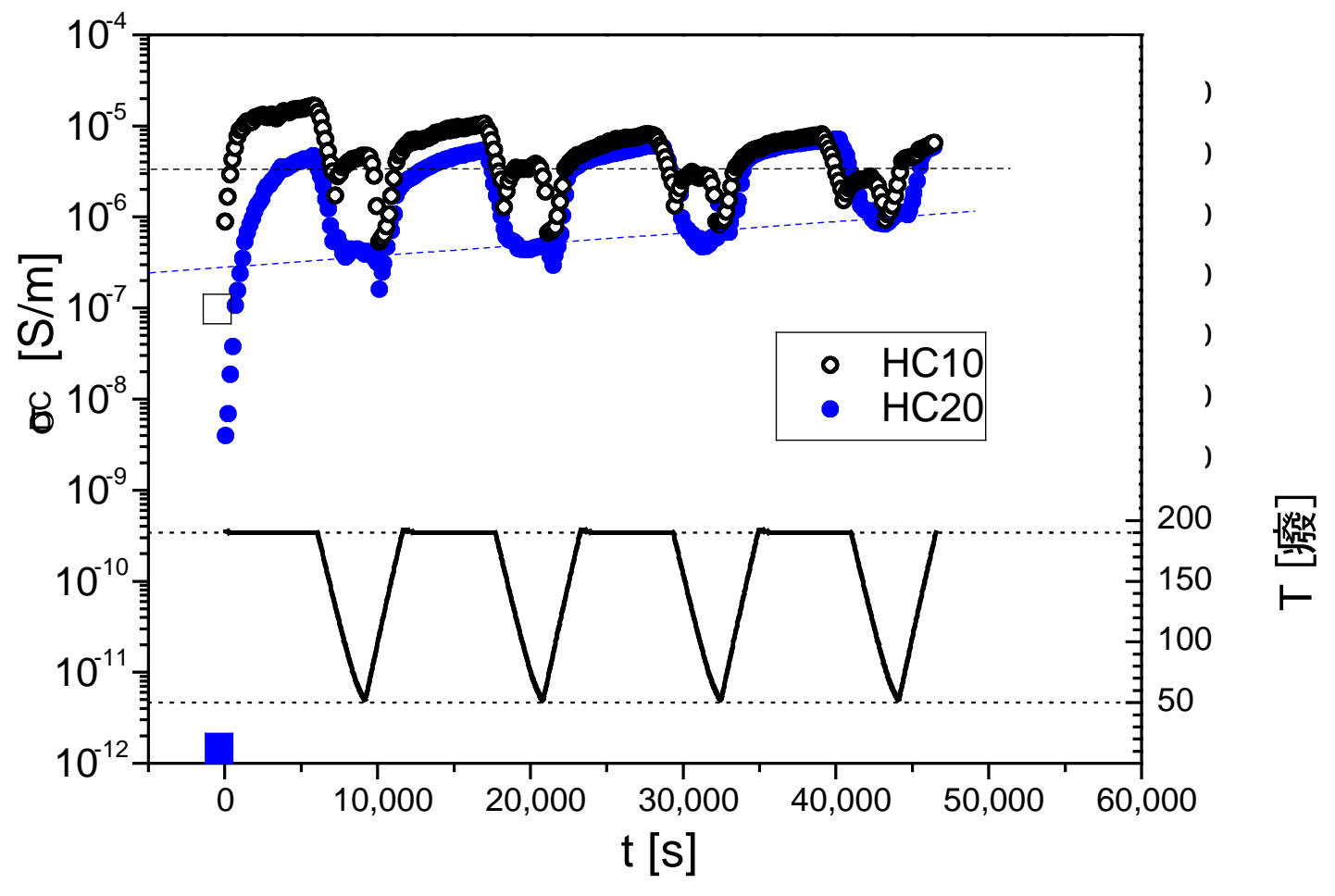

Figure 6. Time dependence of conductivity for the composites HC10 and HC20 during isothermal annealing at $190^{\circ} \mathrm{C}$ and followed-up cooling and heating cycles. The big filled squares at start time are the conductivity of HC10 and HC20 at room temperature (values from figure 3). The temperature program is inserted below.

The DSC melting curves of HC10 and HC20 composites are shown in Figure 7. The single melting single peaks of $\mathrm{HC} 10$ and $\mathrm{HC} 20$, around $130^{\circ} \mathrm{C}$, are attributed to the melting of foldedchain lamellae[22]. This indicates that the average lamellar thickness is almost the same in both composites. It is worth noting that the endset of the melting peak for $\mathrm{HC} 20$ is about $135.5^{\circ} \mathrm{C}$, which is $3.1^{\circ} \mathrm{C}$ higher than that of HC10. From comparison of the two first melting curve, we can see that a part of HC20 crystals (about $10 \%$ in area) melt at higher temperature than overall melting temperature of HC10. This suggests that HC20 crystals are having a small amount of 
thicker kebabs and/or possibly an extended-chain layer wrapping around CNT. After annealing at $190^{\circ} \mathrm{C}$ for 5 min followed by crystallization, we re-melt the sample during a second heating run. The melting peak and the endset of $\mathrm{HC} 20-2 \mathrm{~h}$ shift slightly up by $1.5^{\circ} \mathrm{C}$ and $1{ }^{\circ} \mathrm{C}$, respectively, suggesting formation of slightly thicker crystal lamellae (presumably at the interface with the CNT) and/or an extended-chain layer on the CNT during first heating and cooling cycle.

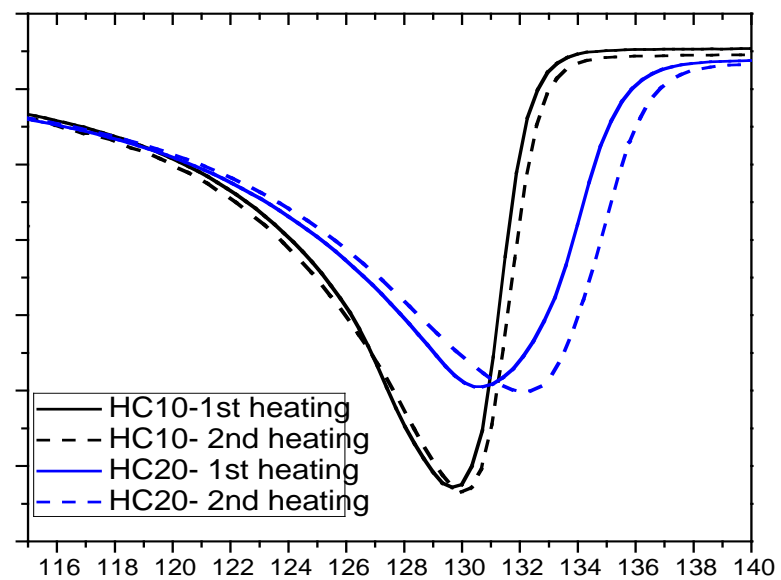

Figure 7. DSC heating curves of $\mathrm{HC} 10$ and $\mathrm{HC} 20$ at a heating rate of $10^{\circ} \mathrm{C} / \mathrm{min}$. $1 \mathrm{~h}$ and $2 \mathrm{~h}$ stands for the 1st and 2nd cycle of heating from 25 to $200^{\circ} \mathrm{C}$, respectively.

In-situ rheo-SAXS is performed to further reveal the difference between HC10 and HC20 on HDPE lamellar arrangement. The experimental details can be found in the experimental section as well as the references[43]. The spacing between the adjacent lamellae (long period, L) is estimated and displayed as a function of temperature during different cycles of heating. The detailed calculation is demonstrated in literature[44]. As shown in Figure 8, for both HC10 and HC20, the value of L increases dramatically above a certain temperature, indicating gradual melting and disappearance of lamellae. The start value of L for HC20-1h and HC10-1h (22 nm) 
is $6 \mathrm{~nm}$ lower in comparison with the other heating curves $(28 \mathrm{~nm})$. This difference is probably caused by the pre-cooling history during hot-press and subsequent cooling.

In general, it takes a higher temperature for HC20 to be fully melted than for HC10. This indirectly suggests that a part of crystals in HC20 is significantly thicker than that of HC10, since we have seen that the average lamellar thickness of these two composites is the same. Moreover, after 3 heating and cooling cycles, the end melting temperature of $\mathrm{HC} 20$ is still about $3^{\circ} \mathrm{C}$ higher. These results are consistent with the above-mentioned DSC data.

The existence of a shish-kebab nanostructure is further verified by TEM images of HDPE/CNT composites after 5 hours of solution extraction of HDPE matrix. As shown in Figure 9(a), the surface of CNT is smooth and no visible HDPE chains are left after Soxhlet extraction, whereas in Figure 9(b) and 9(c), a shish-kebab hybrid structure is found with CNT serving as shish and HDPE lamellae crystals forming as kebab. These TEM pictures are not surprising, since there are many reports regarding the strong interaction between HDPE chains and CNTs. For example, very recently Minus et al.[21] found that the kebabs washed in boiling xylene maintain their molecular orientation although they are mostly rendered amorphous by the washing process. However, the TEM pictures in Figure 9 are simply to prove the existence of shish-kebab structure, as we cannot directly compare the size and thickness of the crystals in TEM due to melting issues caused by high voltage electron emission. 


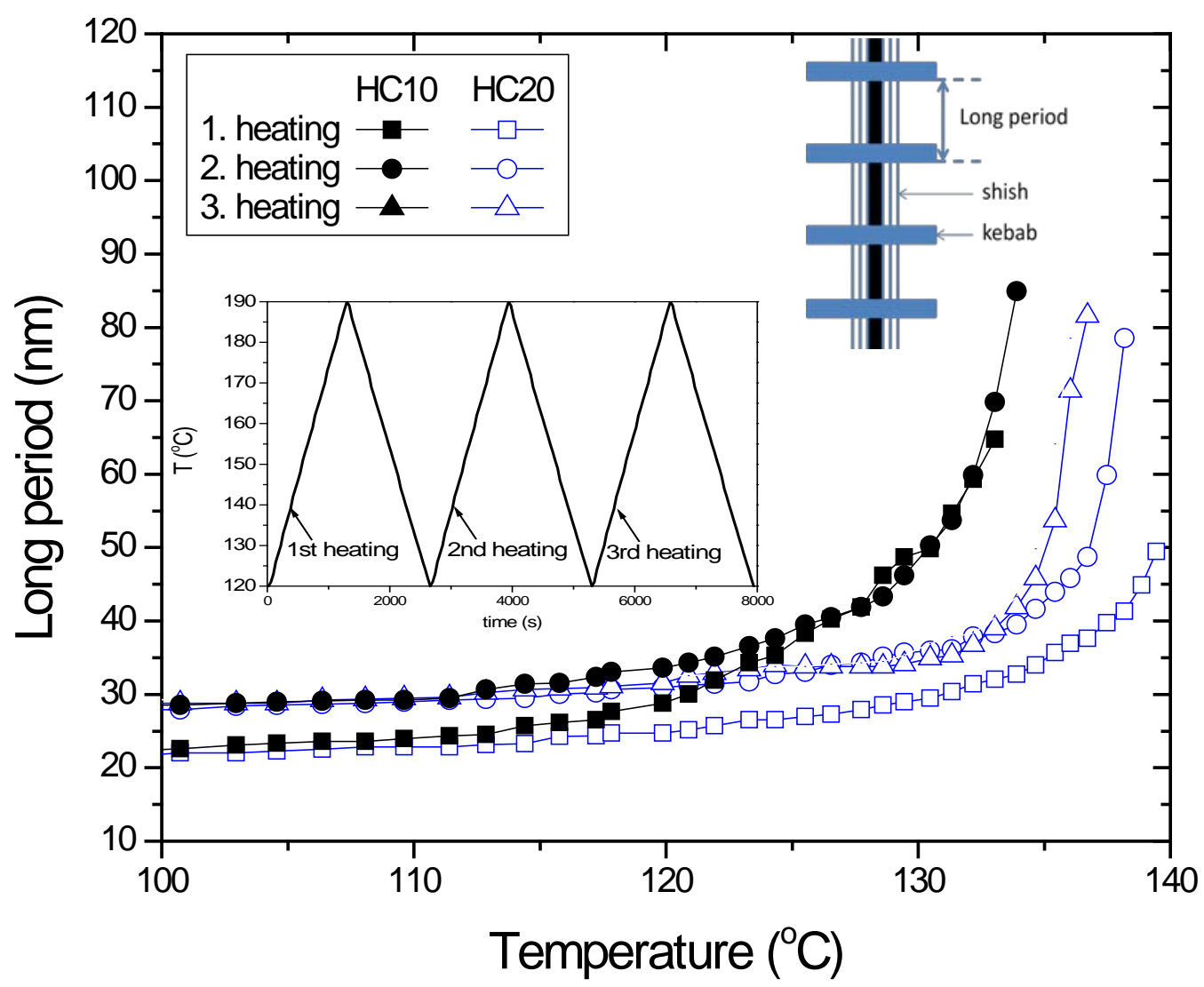

Figure 8. Plots of long period as a function of temperature during heating. The temperature program is inserted.

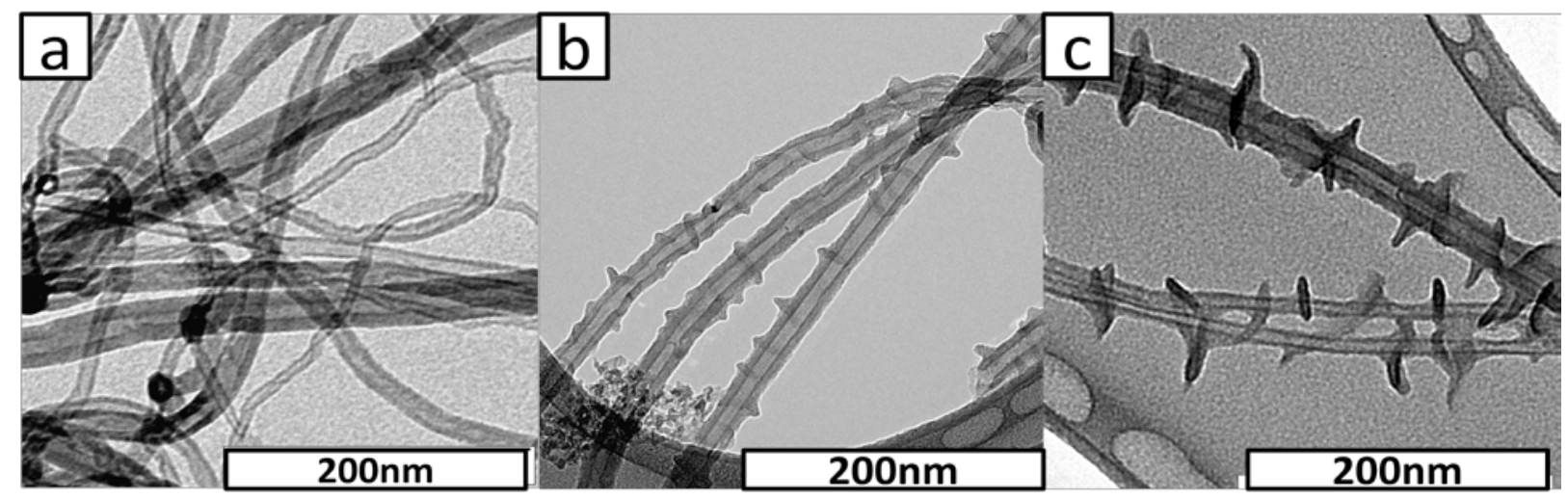

Figure 9. TEM pictures of composites after 5 hours of solution extraction of HDPE matrix. a) pristine CNT solution mixed with HDPE; b) HC10; c) HC20. The background is from holeycarbon films of copper grids. 
It is well-established that the shear flow has a favorable effect on the formation of shish-kebab structure as it can align the PE chains along the surface of CNT. As indicated in the rheological results, the longest relaxation time of HMW-PE is estimated to be more than $10^{4} \mathrm{~s}$, which is the time scale for PE chains orientated along the CNT by shear flow to relax. Moreover, in a CNT/PE composite, strong interfacial adhesion originate from the electrostatic and van der Waal's interactions as well as the epitaxial match between PE chains and CNT. In this context, it is believed that with longer shear-blending time, more of the PE chains are stretched. Therefore, thicker crystals are obtained at the interface of CNT/PE, which block the electrons from hoping from one CNT to another in solid state. Yang et al. has demonstrated that the PE chains are aligned along CNT axis in extended conformations under certain conditions using molecular dynamic simulation ${ }^{[45]}$. This explains very well the shear-mixing time dependence of electrical conductivity and the slow recovery of conductivity with annealing time at $190^{\circ} \mathrm{C}$, which is well above the equilibrium melting temperature of $\mathrm{PE}\left(140^{\circ} \mathrm{C}\right)$. A recently published review paper by Ning et al. ${ }^{[37]}$ summarized the enhanced polymer-filler interfacial interaction via crystallization of polymer chains on the surface of fillers. It has attracted tremendous interest not only due to its crystallography interests in polymer physics, but also due to the fact that it may be a novel strategy to enhance interfacial adhesion and realize the full potential of fillers to reinforce the mechanical performance of the composites. In complement to these studies, we find in this work that the crystallization of polymer chains influenced by shear history may have a significant effect on the electrical conductivity.

\section{Conclusions}

The relationships between melt-mixing time, crystal sizes, and final electrical conductivity of HDPE/CNT nanocomposites have been studied using in-situ rheo-electrical, thermal analysis, 
and small angle synchrotron X-ray scattering (SAXS). For an identical composition, the HDPE/CNT composite melt-mixed for 20 minutes is found to exhibit a more dramatic decrease of conductivity when compared to the same system mixed for only 10 minutes even after annealing at $190^{\circ} \mathrm{C}$ for a long time (>6000s).

This loss of electrical conductivity could not be related to a shortening of CNT as TEM observations clearly evidenced no significant length reduction and damage of the CNT during 10 min and 20 min processing. This phenomenon was neither related to a severe modification of CNT dispersion within the HDPE matrix as TEM analyses confirmed no significant changes for processing time of 10 or 20 minutes.

Electrical properties of the final nanocomposites were found to be greatly connected to HDPE crystallization based on the studies on HMW-PE/CNT composties. Indeed, the presence of CNT was found to affect greatly the nucleation and growth mechanisms of HDPE crystals. The crystal size of HDPE within HDPE/CNT composite processed during 20 minutes is globally larger than that of the same composite melt mixed for only 10 minutes.

\section{Acknowledgements}

The authors thank the European Community for the financial support in the frame of the 7th Framework Program research project “HARCANA”' (Grant Agreement No: NMP3-LA-2008213277). MATERIA NOVA and CIRMAP thanks the "Belgian Federal Government Office

Policy of Science (SSTC)" for general support in the frame of the PAI-6/27 and "Region Wallonne" in the frame of the "Programme d'Excellence : OPTIMAT", and D.A. for the support by the EU FP7 Marie-Curie fellowship “SUPRADYN”. 
We thank Oltea Murariu for the help on melt-mixing and Yoann Paint, Pascale Lipnik, on TEM, and Pascal Simon on dielectrical measurements. We thank Dr. Guido Heunen, Prof. Alain M. Jonas Dr. Wim Pyckhout-Hintzenand Prof. B. Hsiao for the helpful discussions on crystallization and scattering. We also thank FUTURECARBON for kindly supplying the studied CNT.

\section{References:}

1. Iijima S. Nature 1991;354(6348):56-58.

2. Haggenmueller R, Fischer JE, and Winey KI. Macromolecules 2006;39(8):2964-2971.

3. Haggenmueller R, Guthy C, Lukes JR, Fischer JE, and Winey KI. Macromolecules 2007;40(7):2417-2421.

4. Linares A, Canalda JC, Cagiao ME, Garcia-Gutierrez MC, Nogales A, Martin-Gullom I, Vera J, and Ezquerra TA. Macromolecules 2008;41(19):7090-7097.

5. McNally T, Pötschke P, Halley P, Murphy M, Martin D, Bell SEJ, Brennan GP, Bein D, Lemoine P, and Quinn JP. Polymer 2005;46(19):8222-8232.

6. Yang B-X, Pramoda KP, Xu GQ, and Goh SH. Advanced Functional Materials 2007;17(13):2062-2069.

7. Tao F, Bonnaud L, Murariu O, Auhl D, Dubois P, and Bailly C. Macromolecular Chemistry and Physics 2012:doi: 10.1002/macp.201200477.

8. $\quad$ Yu DR and Kim GH. Journal of Applied Polymer Science 2012;124(4):2962-2967.

9. $\quad$ Tang WZ, Santare MH, and Advani SG. Carbon 2003;41(14):2779-2785.

10. Zhang QH, Rastogi S, Chen DJ, Lippits D, and Lemstra PJ. Carbon 2006;44(4):778-785.

11. Verge P, Benali S, Bonnaud L, Minoia A, Mainil M, Lazzaroni R, and Dubois P. European Polymer Journal 2012;48(4):677-683.

12. Min KT and Kim GH. Journal of Applied Polymer Science 2011;120(1):95-100.

13. Trujillo M, Arnal ML, Müller AJ, Laredo E, Bredeau S, Bonduel D, and Dubois P. Macromolecules 2007;40(17):6268-6276.

14. Adhikari AR, Chipara M, and Lozano K. Materials Science and Engineering A 2009;526(1-2):123-127.

15. Barus S, Zanetti M, Bracco P, Musso S, Chiodoni A, and Tagliaferro A. Polymer Degradation and Stability 2010;95(5):756-762.

16. Kanagaraj S, Varanda FR, Zhil'tsova TV, Oliveira MSA, and Simoes JAO. Composites Science and Technology 2007;67(15-16):3071-3077.

17. Lisunova MO, Mamunya YP, Lebovka NI, and Melezhyk AV. European Polymer Journal 2007;43(3):949-958.

18. Li CY, Li LY, Cai WW, Kodjie SL, and Tenneti KK. Advanced Materials 2005;17(9):1198.

19. Li L, Li B, Hood MA, and Li CY. Polymer 2009;50(4):953-965965.

20. Liang S, Wang K, Chen D, Zhang Q, Du R, and Fu Q. Polymer 2008;49(23):492549294929. 
21. Minus ML, Chae HG, and Kumar S. ACS Applied Materials \& Interfaces 2011;4(1):326330.

22. Yang J, Wang $\mathrm{C}$, Wang $\mathrm{K}$, Zhang Q, Chen $\mathrm{F}$, Du R, and Fu Q. Macromolecules 2009;42(18):7016-7023.

23. Alig I, Lellinger D, Dudkin SM, and Pötschke P. Polymer 2007;48(4):1020-1029.

24. $\quad$ Alig I, Skipa T, Lellinger D, and Pötschke P. Polymer 2008;49(16):3524-3532.

25. Pegel S, Pötschke P, Petzold G, Alig I, Dudkin SM, and Lellinger D. Polymer 2008;49(4):974-984.

26. Villmow T, Kretzschmar B, and Pötschke P. Composites Science and Technology 2010;70(14):2045-2055.

27. Hernandez JJ, Garcia-Gutierrez MC, Nogales A, Rueda DR, Kwiatkowska M, Szymczyk A, Roslaniec Z, Concheso A, Guinea I, and Ezquerra TA. Composites Science and Technology 2009;69(11-12):1867-1872.

28. Sahoo NG, Thet NT, Tan QH, Li L, Chan SH, Zhao J, and Yu S. Journal of Nanoscience and Nanotechnology 2009;9(10):5910-5919.

29. Villmow T, Pegel S, Pötschke P, and Wagenknecht U. Composites Science and Technology 2008;68(3-4):777-789.

30. Villmow T, Pötschke P, Pegel S, Häußler L, and Kretzschmar B. Polymer 2008;49(16):3500-3509.

31. Al-Saleh MH and Sundararaj U. Polymers for Advanced Technologies;22(2):246-253.

32. Krause B, Boldt R, and Pötschke P. Carbon 2011;49(4):1243-1247.

33. Krause B, Villmow T, Boldt R, Mende M, Petzold G, and Pötschke P. Composites Science and Technology 2011;71(8):1145-1153.

34. Socher R, Krause B, Müller MT, Boldt R, and Pötschke P. Polymer 2012;53(2):495-504.

35. Patil N, Balzano L, Portale G, and Rastogi S. Carbon 2010;48(14):4116-4128.

36. Patil N, Balzano L, Portale G, and Rastogi S. Macromolecular Chemistry and Physics 2009;210(24):2174-2187.

37. Ning N, Fu S, Zhang W, Chen F, Wang K, Deng H, Q. Z, and Fu Q. Progress in Polymer Science 2012.

38. Keunings R, Bailly C, and van Ruymbeke E. Journal of Non-Newtonian Fluid Mechanics 2005;128(1):7-2222.

39. van Ruymbeke E, Liu C-Y, and Bailly C. Rheology Reviews 2007:53-134.

40. van Ruymbeke E, Stephenne V, Daoust D, Godard P, Keunings R, and Bailly C. Journal of Rheology 2005;49(6):1503-15201520.

41. Stauffer D and Aharony A. Introduction To Percolation Theory. London: CRC Press, 1994.

42. Menzer K, Krause B, Boldt R, Kretzschmar B, Weidisch R, and Pötschke P. Composites Science and Technology 2011;71(16):1936-1943.

43. Struth B, Hyun K, Kats E, Meins T, Walther M, Wilhelm M, and Grubel G. Langmuir 2011;27(6):2880-2887.

44. Chen YH, Zhong GJ, Lei J, Li ZM, and Hsiao BS. Macromolecules 2011;44(20):80808092.

45. Yang H, Chen Y, Liu Y, Cai WS, and Li ZS. Journal of Chemical Physics 2007;127(9):094902/094901. 
